\title{
Cover time of a random graph with given degree sequence
}

\author{
Mohammed Abdullah ${ }^{1}$ and Colin Cooper ${ }^{1}$ and Alan Frieze $\|^{2}$ \\ ${ }^{1}$ Department of Computer Science, King's College, University of London, London WC2R 2LS, UK \\ ${ }^{2}$ Department of Mathematical Sciences, Carnegie Mellon University, Pittsburgh PA15213, USA
}

In this paper we establish the cover time of a random graph $G(\mathbf{d})$ chosen uniformly at random from the set of graphs with vertex set $[n]$ and degree sequence $\mathbf{d}$. We show that under certain restrictions on d, the cover time of $G(\mathbf{d})$ is with high probability asymptotic to $\frac{d-1}{d-2} \frac{\theta}{d} n \log n$. Here $\theta$ is the average degree and $d$ is the effective minimum degree. The effective minimum degree is the first entry in the sorted degree sequence which occurs order $n$ times.

Keywords: Random walks, random graphs, cover time

\section{Introduction}

Let $G=(V, E)$ be a connected graph with $|V|=n$ vertices and $|E|=m$ edges. For $v \in V$, let $C_{v}$ be the expected time taken for a simple random walk $\mathcal{W}_{v}$ on $G$ starting at $v$, to visit every vertex of $G$. The vertex cover time $C_{G}$ of $G$ is defined as $C_{G}=\max _{v \in V} C_{v}$. The vertex cover time of connected graphs has been extensively studied. It is a classic result of [1] that $C_{G} \leq 2 m(n-1)$. It was shown by [6], [7], that for any connected graph $G$, the cover time satisfies $(1-o(1)) n \log n \leq C_{G} \leq(1+o(1)) \frac{4}{27} n^{3}$. Between these two extremal examples, the cover time, both exact and asymptotic, has been determined for a number of different classes of random and non-random graphs. The above bounds being general, we can often gain insight into the behavior of random walks by taking into consideration the structural properties of the underlying graphs, and studying how estimates of the the main parameters - including cover time - are affected in light of this knowledge.

One way is to specify the degrees of the vertices and investigate how far this determines the properties of the walk. For any connected graph, [10] gave a cover time upper bound of $4 n^{2} d_{\text {ave }} / d_{\text {min }}$, where $d_{\text {ave }}$ is the average degree and $d_{\min }$ is the minimum degree. For $d$-regular graphs, [8] improved this to $2 n^{2}$. It is interesting that the vertex degree $d$ does not itself feature in this bound. Although the bound of [10] is clearly $O\left(n^{2}\right)$ when $d_{\text {ave }} / d_{\min }$ is a constant, the factor of $1 / d_{\min }$ means that the result is not robust to small numbers of small degree vertices that should have inconsequential effects on the cover time. The paper [5] addresses this to some extent with a result on cyclic cover time. For an optimal cyclic list of all the vertices of a graph, the cyclic cover time is the expected time it takes a random walk to travel

\footnotetext{
${ }^{\dagger}$ Research supported in part by NSF Grant ccf0502793

1365-8050 @ 2010 Discrete Mathematics and Theoretical Computer Science (DMTCS), Nancy, France
} 
from vertex to vertex along the list, until it completes a full cycle. This is obviously an upper bound on the cover time. The paper [5] show that the cyclic cover time for a graph is $\Theta\left(n^{2} d_{\text {ave }}\left(d^{-1}\right)_{\text {ave }}\right)$, where $\left(d^{-1}\right)_{a v e}$ is the average of the inverse of the degrees. Note, in the case of a graph where the sets of vertices of degree $d$ and $\Delta>>d$ each each make up a constant proportion of the total number of vertices, we get $n^{2} d_{\text {ave }}\left(d^{-1}\right)_{\text {ave }}=\Omega\left(n^{2} \Delta / d\right)$

In this paper we extend the above results by studying the cover time of random graphs $\mathcal{G}(\mathbf{d})$ picked uniformly at random (uar) from the set $\mathcal{G}(\mathbf{d})$ of simple graphs with vertex set $V=[n]$ and degree sequence $\mathbf{d}=\left(d_{1}, d_{2}, \ldots, d_{n}\right)$. We make the following definitions: Let $V_{j}=\left\{i \in V: d_{i}=j\right\}$ and let $n_{j}=\left|V_{j}\right|$. Let $\sum_{i=1}^{n} d_{i}=2 m$ and let $\theta=2 m / n$ be the average degree.

It seems reasonable to ask how the various entries in the degree sequence affect the cover time. In particular, how much does the cover time depend on the vertices of low degree, and how much on the average degree of the graph? In fact, as in [5], both parameters play a part, as is shown in Theorem 1]

Immediately on fixing the degree sequence, $\mathbf{d}$, some definitional problems arise, as e.g. there may be just a few low degree vertices spread over a wide range. To get round this, we define an absolute minimum degree $\delta$, and an effective minimum degree $d$. The effective minimum degree is the first entry in the sorted degree sequence which occurs order $n$ times. We fix the minimum degree at 3 to ensure the graph is connected (whp). Between the minimum degree, and the effective minimum degree, we place an upper bound on the number $n_{i}$ of vertices of degree $i$. The bound we choose of $n_{i}=O\left(n^{c i / d}\right)$, is not as arbitrary as it looks. Certainly when $c=d /(d-1)$, the effective minimum degree drops below $d$, so clearly there is some $c<d /(d-1)$ which is critical. Finally, we make some constraints on the average degree and upper tail of the degree sequence, to ensure simple graphs occur with high enough probability in the configuration model. Thus we are left with the following list of conditions.

Let $0<\alpha<1$ be constant, $0<c<1 / 8$ be constant and let $d$ be a positive integer. Let $\gamma \rightarrow \infty$ with $n$. We suppose the degree sequence $\mathbf{d}$ satisfies the following conditions:

(i) Average degree $\theta=o(\sqrt{\log n})$.

(ii) Minimum degree $\delta \geq 3$.

(iii) For $\delta \leq i<d, n_{i}=O\left(n^{c i / d}\right)$.

(iv) $n_{d}=\alpha n+o(n)$. We call $d$ the effective minimum degree.

(v) Maximum degree $\Delta=O\left(n^{c(d-1) / d}\right)$.

(vi) Upper tail size $\sum_{j=\gamma \theta}^{\Delta} n_{j}=O(\Delta)$.

We call a degree sequence $\mathbf{d}$ which satisfies conditions (i)-(vi) nice, and apply the same adjective to $\mathcal{G}(\mathbf{d})$.

Theorem 1 Let $G(\boldsymbol{d})$ be chosen uar from $\mathcal{G}(\boldsymbol{d})$, where $\boldsymbol{d}$ is nice. Then whp

$$
C(G(\boldsymbol{d})) \sim \frac{d-1}{d-2} \frac{\theta}{d} n \log n .
$$


We note that if $d \sim \theta$, i.e. the graph is pseudo-regular, then

$$
C(G) \sim \frac{d-1}{d-2} n \log n
$$

which extends the result of [3] for random $d$-regular graphs.

\section{Structure of the paper}

The proof of Theorem 1 is based on an application of (5) below. Put simply, (5) says that, if we ignore which vertices the random walk visits during the mixing time, the probability a vertex $v$ is not visited by step $t$ is asymptotic to $\exp \left(-\pi_{v} t / R_{v}\right)$. Here $\pi_{v}=d(v) / 2 m$ and $R_{v}$ is the expected number of returns to $v$ during the mixing time, for a walk starting at $v$. We estimate $R_{v}$ in Section 4 , and describe and prove the required whp graph properties in Section 3 The proof that $(5)$ is valid whp for $\mathcal{G}(\mathbf{d})$ is similar to proofs in earlier papers and is given in the Appendix. The cover time $C(G)$ is established as follows in Section 5. Firstly a general upper bound is proved in Section 5.1 In Section 5.2 a lower bound is determined by the set of vertices $S$ which maximize $\sum_{v \in S} \exp \left(-\pi_{v} t / R_{v}\right)$.

\section{Estimating first visit probabilities}

\section{Convergence of the random walk}

In this section $G$ denotes a fixed connected graph with $n$ vertices. A random walk $\mathcal{W}_{u}$ is started from a vertex $u$. Let $\mathcal{W}_{u}(t)$ be the vertex reached at step $t$, let $P$ be the matrix of transition probabilities of the walk and let $P_{u}^{(t)}(v)=\operatorname{Pr}\left(\mathcal{W}_{u}(t)=v\right)$. We assume that the random walk $\mathcal{W}_{u}$ on $D$ is ergodic with stationary distribution $\pi$, where $\pi_{v}=d(v) /(2 m)$, and $d(v)$ is the degree of vertex $v$.

Let

$$
d(t)=\max _{u, x \in V}\left|P_{u}^{(t)}(x)-\pi_{x}\right|,
$$

and let $T$ be a positive integer such that for $t \geq T$

$$
\max _{u, x \in V}\left|P_{u}^{(t)}(x)-\pi_{x}\right| \leq n^{-3} .
$$

Fix two vertices $u, v$. Considering the walk $\mathcal{W}_{v}$, starting at $v$, let $r_{t}=\operatorname{Pr}\left(\mathcal{W}_{v}(t)=v\right)$ be the probability that this walk returns to $v$ at step $t=0,1, \ldots$. Let

$$
R_{T}(z)=\sum_{j=0}^{T-1} r_{j} z^{j}
$$

and

$$
\lambda=\frac{1}{K T}
$$

for a sufficiently large constant $K$.

For $t \geq T$ let $\boldsymbol{A}_{v}(t)$ be the event that $\mathcal{W}_{u}$ does not visit $v$ in steps $T, T+1, \ldots, t$. 
Lemma 2 Suppose that

(a) For some constant $\psi>0$, we have

$$
\min _{|z| \leq 1+\lambda}\left|R_{T}(z)\right| \geq \psi
$$

(b) $T^{2} \pi_{v}=o(1)$ and $T \pi_{v}=\Omega\left(n^{-2}\right)$ for all $v \in V$.

There exists

$$
p_{v}=\frac{\pi_{v}}{R_{v}\left(1+O\left(T \pi_{v}\right)\right)}
$$

where

$$
R_{v}=R_{T}(1)
$$

is from (2), such that for all $v \in V$ and $t \geq T$,

$$
\operatorname{Pr}\left(\boldsymbol{A}_{v}(t)\right)=\frac{\left(1+O\left(T \pi_{v}\right)\right)}{\left(1+p_{v}\right)^{t}}+O\left(T^{2} \pi_{v} e^{-\lambda t / 2}\right) .
$$

\section{Required graph properties}

\subsection{Mixing time}

Given a graph $G$, the conductance $\Phi(G)$ of a random walk $\mathcal{W}_{u}$ on $G$ is defined by

$$
\Phi(G)=\min _{\pi(S) \leq 1 / 2} \frac{e(S: \bar{S})}{d(S)}
$$

where $d(S)=\sum_{i \in S} d_{i}$, and $e(A: B)$ denotes the number of edges with one endpoint in $A$ and the other in $B$. The lemma below follows from Lemma 10 of the Appendix by applying 8 .

Lemma 3 Let $\boldsymbol{d}$ be a nice degree sequence and let $G(\boldsymbol{d})$ be chosen uniformly at random from the $\mathcal{G}(\boldsymbol{d})$, then whp

$$
\Phi(G) \geq 0.01 .
$$

Note that $\Phi(G) \geq 0.01$ in Lemma 3 implies $G(\mathbf{d})$ is connected.

We note a result from Sinclair [11], that

$$
\left|P_{u}^{(t)}(x)-\pi_{x}\right| \leq\left(\pi_{x} / \pi_{u}\right)^{1 / 2}\left(1-\Phi^{2} / 2\right)^{t} .
$$

Referring to Lemma 3 and (6), if we choose $A$ sufficiently large and

$$
T=A \log n
$$

then (1) holds.

There is a technical point here. The result (6) assumes that the walk is lazy. A lazy walk moves to a neighbour with probability $1 / 2$ at any step. This assumption halves the conductance. Asymptotically, the cover time, and the value of $R_{T}(1)$ are also doubled. Otherwise, the lazy assumption has a negligible effect on the analysis. We will ignore this assumption for the rest of the paper, and continue as though there are no lazy steps. 


\subsection{Structural properties of $G(\boldsymbol{d})$}

We make our calculations in the configuration model, see Bollobás [2]. Let $W=[2 m]$ be our set of configuration points and let $W_{i}=\left[d_{1}+\cdots+d_{i-1}+1, d_{1}+\cdots+d_{i}\right], i \in[n]$, partition $W$. The function $\phi: W \rightarrow[n]$ is defined by $w \in W_{\phi(w)}$. Given a pairing $F$ (i.e. a partition of $W$ into $m$ pairs) we obtain a (multi-)graph $G_{F}$ with vertex set $[n]$ and an edge $(\phi(u), \phi(v))$ for each $\{u, v\} \in F$. Choosing a pairing $F$ uniformly at random from among all possible pairings of the points of $W$ produces a random (multi-)graph $G_{F}$. Let $\nu=\sum_{i} d_{i}\left(d_{i}-1\right) /(2 m)$. Assuming that $\Delta=o\left(m^{1 / 3}\right)$ (as it will be for nice sequences), the probability that $G_{F}$ is simple is given by

$$
P_{S}=\operatorname{Pr}\left(G_{F} \text { is simple }\right) \sim e^{-\frac{\nu}{2}-\frac{\nu^{2}}{4}},
$$

(see e.g. [9]), and each simple graph $G \in \mathcal{G}(\mathbf{d})$ is equiprobable.

Observe that our assumptions (i)-(vi) that $\mathbf{d}$ is nice imply that $\nu=o(\sqrt{\log n})$. Indeed if $\theta=\sqrt{\log n} / \gamma^{3}$ where $\gamma \rightarrow \infty$ then

$$
\nu \leq \frac{1}{\theta n}\left(\sum_{j=3}^{\gamma \theta} n_{j} j^{2}+\sum_{j=\gamma \theta}^{\Delta} n_{j} j^{2}\right) \leq \frac{1}{\theta n}\left(n \gamma^{2} \theta^{2}+O\left(\Delta^{3}\right)\right)=o(\sqrt{\log n}) .
$$

All the whp statements in this paper fail with probability at most $n^{-\Omega(1)}$, whereas $P_{S}$ in 8 is at least $e^{-o(\log n)}$. This justifies our use of the configuration model.

Let $C$ be a large constant and let

$$
\omega=\log \log \log n, \quad \omega^{\prime}=C \log \log n .
$$

We use these values for $\omega, \omega^{\prime}$ throughout the paper. A cycle $C$ or path $P$ is small, if it has at most $2 \omega^{\prime}+1$ vertices, otherwise it is large.

Let

$$
\ell=B \log ^{2} n
$$

for some large constant $B$. A vertex $v$ is light if it has degree at most $\ell$, otherwise it is heavy. A small path is light if all vertices are light. A small cycle is light if it has at most one heavy vertex.

For a vertex $v$, let $G_{v}$ be the subgraph induced by the set of vertices within a distance $\omega$ of $v$. A vertex $v$ is locally tree-like if $G_{v}$ is a tree. A vertex $v$ is $r$-regular, if it is locally tree-like and each vertex of $G_{v}$, (with the possible exception of $v$ ), has degree $r$. A vertex $v$ is $r$-compliant, if there exists a tree subgraph $\mathcal{T}_{v}$ of $G_{v}$ rooted at $v$, in which each vertex of $\mathcal{T}_{v}$ (with the possible exception of $v$ ) has degree $r$.

\section{Lemma 4 Whp:}

(a) No pair of small light cycles are connected by a small light path.

(b) No pair of vertices on a small light cycle are joined by a small light path.

Proof We first note a useful inequality. For integer $x>0$, let $F(2 x)=\frac{(2 x) !}{2^{x} x !}$, then

$$
\frac{F(\theta n-2 x)}{F(\theta n)}=\frac{(\theta n-2 x) !}{\left(\frac{\theta n}{2}-x\right) ! 2^{\frac{\theta n}{2}-x}} \frac{\left(\frac{\theta n}{2}\right) ! 2^{\frac{\theta n}{2}}}{(\theta n) !}=\left(\prod_{i=1}^{x} \theta n-2 i+1\right)^{-1} \leq\left(\frac{1}{\theta n-2 x+1}\right)^{x} .
$$


We prove part (a) in detail; the calculations for part (b) are similar.

(a) Let $\mu$ denote the expected number of light cycle-path-cycle subgraphs consisting of cycles of length $a, b$ joined by a path length $c$. Then

$$
\mu \leq \sum_{a=3}^{2 \omega^{\prime}+1} \sum_{b=3}^{2 \omega^{\prime}+1} \sum_{c=1}^{2 \omega^{\prime}+1}\left(\begin{array}{l}
n \\
a
\end{array}\right)\left(\begin{array}{l}
n \\
b
\end{array}\right)\left(\begin{array}{l}
n \\
c
\end{array}\right) \frac{(a-1) !}{2} \frac{(b-1) !}{2} c ! a b \ell^{2(a+b+c-2)} \Delta^{6} \frac{\mathcal{F}(\theta n-2(a+b+c+1))}{\mathcal{F}(\theta n)}
$$

Explanation. Choose $a$ vertices for one cycle, $b$ vertices for the other and $c$ vertices for the path. At most one vertex in a cycle is not light, and has degree more than $\ell$ (and at most $\Delta$ ). Each light vertex has up to $\ell(\ell-1)$ ways to connect to a neighbour, for a total of (at least) $((a-1)+(b-1)+c)$ light vertices, explaining the exponent of $\ell$. The remaining, possibly heavy vertex in each cycle can connect in up to $\Delta(\Delta-1)$ ways to neighbours in the cycle and $\Delta-2$ ways from a cycle to a path. Thus $\mu$ is bounded by

$$
\begin{aligned}
\mu & =\sum_{a=3}^{2 \omega^{\prime}+1} \sum_{b=3}^{2 \omega^{\prime}+1} \sum_{c=1}^{2 \omega^{\prime}+1} n^{a} n^{b} n^{c} \ell^{2(a+b+c-2)} \Delta^{6}\left(\frac{1}{\theta n-12 \omega^{\prime}+6}\right)^{a+b+c} \\
& \leq \frac{\Delta^{6}}{\theta n-12 \omega^{\prime}+6} \sum_{a} \sum_{b} \sum_{c}\left(\frac{n \ell^{2}}{\theta n-12 \omega^{\prime}+6}\right)^{a+b+c} \\
& =O\left(\frac{\Delta^{6} \ell^{12 \omega^{\prime}+6} \omega^{\prime 3}}{\theta n}\right)
\end{aligned}
$$

Thus $\operatorname{Pr}(\mu>0)=o\left(n^{-\epsilon}\right)$, for some constant $\epsilon>0$, since $\Delta=O\left(n^{c(d-1) / d}\right)$ where $c<1 / 6$.

\section{Lemma 5 Whp:}

(a) The number of vertices $v \in V$ that are not $d$-compliant is at most $n^{4 c(d-1) / d}$.

(b) There is no small vertex $v, \delta \leq d(v)<d$ which is not d-compliant.

Proof (a) We lower bound the probability $P$ that $v$ is $d$-compliant by the success, in the configuration model, of the following process.

Process $\mathcal{P}$ : For $0 \leq i \leq \omega-1$, and for each vertex $w$ at level $i$, the first $d-1$ unpaired points of $w$ pair with points of distinct unused vertices $u$ of degree $d(u) \geq d$.

The tree created by process $\mathcal{P}$ involves $N_{1}-1=d_{v} \sum_{i=0}^{i=\omega-1}(d-1)^{i} \leq \Delta(d-1)^{\omega}$ pairings. Let $\sigma$ represent the sum of degrees of vertices of degree less than $d$. Thus

$$
P \geq \prod_{i=1}^{N_{1}} \frac{\theta n-i \Delta-\sigma}{\theta n-2 i+1} \geq\left(1-\frac{N_{1} \Delta+\sigma}{\theta n}\right)^{N_{1}}
$$

Let $X$ count the number of vertices $v$ that are not $d$-compliant. Using the inequality $1-(1-x)^{y} \leq x y$ for real $x, y, 0 \leq x \leq 1, y \geq 1$, we have

$$
\mathbf{E}[X] \leq n(1-P)=n\left(1-\left(1-\frac{N_{1} \Delta+\sigma}{\theta n}\right)^{N_{1}}\right) \leq \frac{N_{1}\left(N_{1} \Delta+\sigma\right)}{\theta} .
$$


We have that $\Delta=O\left(n^{c(d-1) / d}\right)$, and from $n_{i}=O\left(n^{c i / d}\right)$ we find that $\sigma=O\left(n^{c(d-1) / d}\right)$. Thus

$$
E[X]=\tilde{O}\left(\Delta^{3}+\Delta \sigma\right)=\tilde{O}\left(n^{3 c(d-1) / d}\right) \leq K \log ^{L} n n^{3 c(d-1) / d}
$$

for some $K, L>0$. Then,

$$
\operatorname{Pr}\left(X \geq n^{4 c(d-1) / d}\right)=\tilde{O}\left(n^{-c(d-1) / d}\right)
$$

(b) In this case we have that the number of small vertices is $O\left(n^{\frac{c(d-1)}{d}}\right)$ and so $\mathbf{E}[X]=\tilde{O}\left(n^{\frac{2 c(d-1)}{d}-1}\right)$.

Lemma 6 Whp: There are $n^{1-o(1)} d$-regular vertices $v \in V$ with $d(v)=d$.

Proof We consider $d$-regular vertices that have a root vertex $v$ of degree $d$. Recall that $n_{d}=\left|V_{d}\right|=$ $\alpha n+o(n)$ for some constant $\alpha>0$. Let $N_{2}=1+d(d-1)^{\omega}$. A $d$-regular tree of depth $\omega$ contains $N_{2}$ vertices. We proceed in a similar manner to Lemma 5 and bound the probability $P$ that a vertex $v$ is $d$-regular by bounding the probability of success of the construction of a $d$-regular tree in the configuration model.

$$
P=\operatorname{Pr}(\text { a vertex } v \text { is } d \text {-regular })=\prod_{i=1}^{N_{2}-1} \frac{d\left(n_{d}-i\right)}{\theta n-2 i+1} \geq\left(d \frac{n_{d}-N_{2}}{\theta n}\right)^{N_{2}} .
$$

Let $M$ count the number of $d$-regular vertices, then $\mathbf{E}[M]=\mu=n_{d} P$, and

$$
\mu=\mathbf{E}[M] \geq n^{1-o(1)} .
$$

To estimate $\operatorname{Var}[M]$, let $I_{v}$ be the indicator that vertex $v$ is $d$-regular. We have

$$
\mathbf{E}\left[M^{2}\right]=\mu+\sum_{v \in V_{d}} \sum_{w \in V_{d}, w \neq v} \mathbf{E}\left[I_{v} I_{w}\right]
$$

and

$$
\mathbf{E}\left[I_{v} I_{w}\right]=\operatorname{Pr}\left(v, w \text { are } d \text {-regular, } G_{v} \cap G_{w}=\emptyset\right)+\operatorname{Pr}\left(v, w \text { are } d \text {-regular, } G_{v} \cap G_{w} \neq \emptyset\right) .
$$

Now

$$
\operatorname{Pr}\left(v, w \text { are } d \text {-regular, } G_{v} \cap G_{w}=\emptyset\right)=\prod_{i=1}^{2 N_{2}-2} \frac{d\left(n_{d}-i-1\right)}{\theta n-2 i+1} \leq P^{2} .
$$

For any vertex $v$, the number of vertices $u$ such that $G_{v} \cap G_{w} \neq \emptyset$ is bounded from above by $N_{2}+d N_{2}^{2}$. Using this and (17), we can bound 16 from above by $\mu+\mu^{2}+\mu\left(N_{2}+d N_{2}^{2}\right)$.

By the Chebychev Inequality, for some constant $0<\tilde{\epsilon}<1$,

$$
\operatorname{Pr}\left(|M-\mu|>\mu^{\frac{1}{2}+\tilde{\epsilon}}\right) \leq \frac{\operatorname{Var}[M]}{\mu^{1+2 \tilde{\epsilon}}}=\frac{\mathbf{E}\left[M^{2}\right]-\mathbf{E}[M]^{2}}{\mu^{1+2 \tilde{\epsilon}}} \leq \frac{\mu+\mu N_{2}+\mu d N_{2}^{2}}{\mu^{1+2 \tilde{\epsilon}}}=O\left(n^{-\epsilon}\right) .
$$

The lemma now follows from (15). 


\section{Expected number of returns in the mixing time}

The local graph $\Gamma_{v}$. For vertex $v$, inductively define a sub-graph $\Gamma_{v}$ of $G_{v}$ as follows: If $u \in G_{v}$ is heavy, delete an edge $(u, w) \in G_{v}$ iff there is no $(w, v)$-path that is light. After this process is completed, let $\Gamma_{v}$ be the connected component of $G_{v}$ rooted at $v$. The following lemma is a consequence of this construction.

Lemma 7 Either $\Gamma_{v}$ is a tree, or $\Gamma_{v}$ contains a unique light cycle $C$.

Denote by $\Gamma_{v}^{\circ}$ the subset of the vertices of $\Gamma_{v}$ consisting of pruned heavy vertices, and vertices at distance $\omega$ from the root $v$.

Lemma 8 Let $\mathcal{W}_{v}^{*}$ denote the walk on $\Gamma_{v}$ starting at $v$ with $\Gamma_{v}^{\circ}$ made into absorbing states. Let $R_{v}^{*}=$ $\sum_{t=0}^{\infty} r_{t}^{*}$ where $r_{t}^{*}$ is the probability that $\mathcal{W}_{v}^{*}$ is at vertex $v$ at time $t$. There exists a constant $\zeta \in(0,1)$ such that

$$
R_{v}=R_{v}^{*}+O\left(\zeta^{\omega}\right) .
$$

Proofs of a lemma similar to Lemma 8 are given in e.g. [3]. For completeness the proof of Lemma 8 is given in the Appendix.

Lemma 9 Let $G(\boldsymbol{d})$ be good. For a vertex $v \in V$,

(a) If $v$ is $d$-regular, then $R_{v}=\frac{d-1}{d-2}+O\left(\zeta^{\omega}\right)$.

(b) If $v$ is d-compliant then $R_{v} \leq \frac{d-1}{d-2}(1+o(1))$.

(c) For any $v, R_{v} \leq \frac{\delta-1}{\delta-2}(1+o(1))$.

(a) We calculate $R_{v}^{*}$ for a walk $\mathcal{W}_{v}^{*}$ on an $d$-regular tree $\Gamma_{v}$ with $\Gamma_{v}^{\circ}$ made into absorbing states. For a biased random walk on $(0,1, \ldots, k)$, starting at vertex 1 , with absorbing states $0, k$, and with transition probabilities at vertices $(1, \ldots, k-1)$ of $q=\operatorname{Pr}$ (move left), $p=\operatorname{Pr}$ (move right); then

$$
\operatorname{Pr}(\text { absorption at } k)=\frac{(q / p)-1}{(q / p)^{k}-1} \text {. }
$$

We project $\mathcal{W}_{v}^{*}$ onto $(0,1, \ldots, \omega)$ with $p=\frac{d-1}{d}$ and $q=\frac{1}{d}$ giving

$$
\operatorname{Pr}\left(\text { absorption at } \Gamma_{v}^{\circ}\right)=\left(1-\frac{1}{d-1}\right)\left(1+O\left(\frac{1}{(d-1)^{\omega}}\right)\right) .
$$

Let $f_{v}$ be the probability of a return to $v$. Then

$$
R_{v}^{*}=\frac{1}{1-f_{v}}=\frac{d-1}{d-2}+O\left(\frac{1}{(d-1)^{\omega}}\right)
$$

and part (a) of the lemma follows.

(b) If $v$ is $d$-compliant, we can prune $G_{v}$ removing edges from each vertex (other than $v$ ) until $v$ is $d$-regular. Treating the edges as having unit resistance, this pruning process cannot decrease the effective 
resistance between $v$ and a hypothetical vertex $\zeta$ that is connected by a zero-resistance edge to each of the vertices in $\Gamma_{v}^{\circ}$ (and no others). Then by part (a) and Rayleigh's monotonicity law part (b) of the lemma follows. (Here we are using the the fact that the probability of reaching $\zeta$ before returning to $v$ is equal to $\frac{1}{d(v) R}$ where $R$ is the effective resistance between $v$ and $\zeta$. Rayleigh's Law states that deleting edges increases $R$ ).

(c) All vertices on a path from $v$ to $\Gamma_{v}^{\circ}$ have degree at least $\delta$. Thus in expectation there are at most $(\delta-1) /(\delta-2)+o(1)$ returns to $v$ before absorption. If absorption is at distance $\omega$, the arguments in (a), (b) above apply. If not, absorption is at a heavy vertex $u \in \Gamma_{v}^{\circ}$, that is at distance less then $\omega$ from $v$. Such a vertex will have at most two paths back (on the unique light cycle). All other paths to $v$ in $G(\mathbf{d})$ go via other heavy vertices. Hence if a particle is at $u$, with probability at most $2 / \ell$ it will enter a path to $v$ in $\Gamma_{v}$ and probability at least $1-2 / \ell$ enter a path in which it will only reach $v$ by going through another vertex in $\Gamma_{v}^{\circ}$ first. Thus the probability of reaching $v$ in time $T$ after having visited a heavy vertex in $\Gamma_{v}^{\circ}$ is at most $O(T / \ell)$. So $\sum_{t=0}^{\omega} r_{t}-r_{t}^{*}=O(\omega T / l)=o(1)$.

\section{Cover time of $G(\mathbf{d})$}

\subsection{Upper bound on cover time}

Let $T_{G}(u)$ be the time taken by the random walk $\mathcal{W}_{u}$ to visit every vertex of a connected graph $G$. Let $U_{t}$ be the number of vertices of $G$ which have not been visited by $\mathcal{W}_{u}$ at step $t$. We note the following:

$$
\begin{aligned}
C_{u}=\mathbf{E}\left[T_{G}(u)\right] & =\sum_{t>0} \operatorname{Pr}\left(T_{G}(u) \geq t\right), \\
\operatorname{Pr}\left(T_{G}(u) \geq t\right)=\operatorname{Pr}\left(T_{G}(u)>t-1\right) & =\operatorname{Pr}\left(U_{t-1}>0\right) \leq \min \left\{1, \mathbf{E}\left[U_{t-1}\right]\right\} .
\end{aligned}
$$

Recall from (5) that $\boldsymbol{A}_{s}(v)$ is the event that vertex $v$ has not been visited by time $s$. It follows from 20, (21) that

$$
C_{u} \leq t+1+\sum_{s \geq t} \mathbf{E}\left[U_{s}\right]=t+1+\sum_{v} \sum_{s \geq t} \operatorname{Pr}\left(\boldsymbol{A}_{s}(v)\right)
$$

Let $t_{0}=\left(\frac{d-1}{d-2} \frac{\theta}{d}\right) n \log n$ and $t_{1}=(1+\epsilon) t_{0}$, were $\epsilon=o(1)$ is sufficiently large that all inequalities claimed below hold. We will use the notation $d_{v}$ for $d(v)$. We assume that the high probability claims of Sections 3,4 hold. In the Appendix, we establish that condition (a) of Lemma 2 holds. Condition (b) of Lemma 2, that $T \pi_{v}=o(1)$, holds trivially as the maximum degree is $n^{a}, a<1$.

Recall from (4) that $p_{v}=\left(1+\left(T \pi_{v}\right)\right) d_{v} /\left(\theta n R_{v}\right)$. Thus by (5), the probability that $\mathcal{W}_{u}$ has not visited $v$ during $[T, t]$ is given by

$$
\begin{aligned}
\operatorname{Pr}\left(\boldsymbol{A}_{t}(v)\right) & =(1+o(1)) e^{-t p_{v}}+O\left(T^{2} \pi_{v} e^{-\lambda t / 2}\right) \\
& =(1+o(1)) e^{-t p_{v}}
\end{aligned}
$$

Thus

$$
\begin{aligned}
\sum_{t \geq t_{1}}(1+o(1)) e^{-t p_{v}} & =(1+o(1)) e^{-t_{1} p_{v}} \sum_{t \geq t_{1}} e^{-\left(t-t_{1}\right) p_{v}} \\
& \leq 2 p_{v}^{-1} e^{-t_{1} p_{v}} \\
& =O(1) \frac{\theta n R_{v}}{d_{v}} \exp \left\{-(1+\Theta(\epsilon)) \frac{d_{v}}{d} \frac{d-1}{d-2} \frac{\log n}{R_{v}}\right\} .
\end{aligned}
$$


We consider the following partition of $V$ :

(i) $V_{A}=\bigcup_{\delta \leq i<d} V_{i}$.

(ii) $V_{B}=\bigcup_{i \geq \delta}^{\leq}\left\{v \in V_{i}: v\right.$ is $d$-compliant $\}$.

(iii) $V_{C}=\bigcup_{i \geq \delta}\left\{v \in V_{i}: v\right.$ is not $d$-compliant $\}$.

Case (i): $\delta \leq d_{v}<d$.

For these vertices, $\Gamma_{v}$ is $d$-compliant by Lemma 5 . Consider vertices in $V_{i}, i<d$. By Lemma 9 (b), $\left.\left.R_{v} \leq(1+o) 1\right)\right) \frac{d-1}{d-2}$ so for $v \in V_{i}\left[25\right.$ is bounded by $O(\theta n) n^{-(1+o(1)) \frac{i}{d}}$. Recall that $\left|V_{i}\right|=O\left(n^{c i / d}\right)$ where $c<1$. Thus

$$
\sum_{v \in V_{i}} \sum_{t \geq t_{1}}(1+o(1)) e^{-t p_{v}} \leq O(\theta n) n^{c i / d} n^{-(1+o(1)) i / d}=o\left(t_{1}\right) .
$$

Case (ii): $d \leq d_{v}$, $v$ is $d$-compliant.

For $v \in V_{B} \overline{25}$ is bounded by $O(\theta) n^{-\Theta(\epsilon)}$. Therefore

$$
\sum_{v \in V_{B}} \sum_{t \geq t_{1}}(1+o(1)) e^{-t p_{v}} \leq \sum_{v \in V_{B}} O(\theta) n^{-\Theta(\epsilon)}=O(\theta n) n^{-\Theta(\epsilon)}=o\left(t_{1}\right)
$$

Case (iii): $d \leq d_{v}$, $v$ is not $d$-compliant.

For vertices $v \in V_{C}(25)$ is bounded by $O(\theta n) n^{-(1+\Theta(\epsilon)) \frac{\delta-2}{\delta-1} \frac{d-1}{d-2}}$. By Lemma $5,\left|V_{C}\right| \leq n^{4 c(d-1) / d}$ where $4 c<1 / 2 \leq \frac{d}{d-2} \frac{\delta-2}{\delta-1}$. Hence

$$
\begin{aligned}
\sum_{v \in V_{C}} \sum_{t \geq t_{1}}(1+o(1)) e^{-t p_{v}} & =\sum_{v \in V_{C}} O(\theta n) n^{-(1+\Theta(\epsilon)) \frac{\delta-2}{\delta-1} \frac{d-1}{d-2}} \\
& =O\left(n^{c(d-1) / d} \theta n\right) n^{-(1+\Theta(\epsilon)) \frac{\delta-2}{\delta-1} \frac{d-1}{d-2}} \\
& =o\left(t_{1}\right) .
\end{aligned}
$$

In each of the cases above, the term $\sum_{v} \sum_{s \geq t} \operatorname{Pr}\left(\boldsymbol{A}_{s}(v)\right)=o\left(t_{1}\right)$ and thus, from [22), $C_{u} \leq(1+o(1)) t_{1}$ as required. This completes the proof of the upper bound on cover time of $G(\mathbf{d})$.

\subsection{Lower bound on cover time}

Let $t_{2}=(1-\epsilon) t_{0}$, were $\epsilon=o(1)$ is sufficiently large that all inequalities claimed below hold. For vertex $u$ of degree $d$, we exhibit a set of vertices $S$ such that at time $t_{2}$ the probability the set $S$ is covered by the walk $\mathcal{W}_{u}$ tends to zero. Hence $T_{G}(u)>t_{2}$, whp which implies that $C_{G} \geq t_{0}-o\left(t_{0}\right)$.

We construct $S$ as follows. Let $S_{d}$ be the set of $d$-regular vertices of degree $d$. Lemma 6 tells us that $\left|S_{d}\right|=n^{1-o(1)}$. Let $\omega^{\prime}=C \log \log n$ for some large $C$. Let $S$ be a maximal subset of $S_{d}$ such that the distance between any two elements of $S$ is least $\omega^{\prime}$. Thus $|S|=\Omega\left(n^{1-o(1)} / d^{\omega^{\prime}}\right)$.

Let $S(t)$ denote the subset of $S$ which has not been visited by $\mathcal{W}_{u}$ after step $t$. Let $v \in S$, then

$$
\operatorname{Pr}\left(\boldsymbol{A}_{v}\left(t_{2}\right)\right)=(1+o(1)) e^{-t_{2} p_{v}\left(1-O\left(p_{v}\right)\right)}+o\left(n^{-2}\right) .
$$

Hence

$$
\begin{aligned}
\mathbf{E}\left(\left|S\left(t_{2}\right)\right|\right) & \geq(1+o(1))|S| e^{-(1-\epsilon) t_{0} p_{v}} \\
& =\Omega\left(\frac{n^{\epsilon / 2-o(1)}}{d^{\omega^{\prime}}}\right) \rightarrow \infty
\end{aligned}
$$


Let $Y_{v, t}$ be the indicator for the event $\boldsymbol{A}_{t}(v)$. Let $Z=\{v, w\} \subset S$. We will show (below) that that for $v, w \in S$

$$
\mathbf{E}\left(Y_{v, t_{2}} Y_{w, t_{2}}\right)=\frac{1+O\left(T \pi_{v}\right)}{\left(1+p_{Z}\right)^{t_{2}}}+o\left(n^{-2}\right)
$$

where $p_{Z} \sim p_{v}+p_{w}+o(1 / \log n)$. Thus

$$
\mathbf{E}\left(Y_{v, t_{2}} Y_{w, t_{2}}\right)=(1+o(1)) \mathbf{E}\left(Y_{v, t_{2}}\right) \mathbf{E}\left(Y_{w, t_{2}}\right)
$$

which implies

$$
\mathbf{E}\left(\left|S\left(t_{2}\right)\right|\left(\left|S\left(t_{2}\right)\right|-1\right)\right) \sim \mathbf{E}\left(\left|S\left(t_{2}\right)\right|\right)\left(\mathbf{E}\left(\left|S\left(t_{2}\right)\right|\right)-1\right) .
$$

It follows from (27) and (29), that

$$
\operatorname{Pr}\left(S\left(t_{2}\right) \neq \emptyset\right) \geq \frac{\mathbf{E}\left(\left|S\left(t_{2}\right)\right|\right)^{2}}{\mathbf{E}\left(\left|S\left(t_{2}\right)\right|^{2}\right)}=\frac{1}{\frac{\mathbf{E}\left(\left|S\left(t_{2}\right)\right|\left(\left|S\left(t_{2}\right)\right|-1\right)\right)}{\mathbf{E}\left(\left|S\left(t_{2}\right)\right|\right)^{2}}+\mathbf{E}\left(\left|S\left(t_{2}\right)\right|\right)^{-1}}=1-o(1) .
$$

Proof of 28. Let $\widehat{G}$ be obtained from $G$ by merging $v, w$ into a single node $Z$. This node has degree $2 d$ and is $d$-regular. $R_{Z}=\left(R_{v}+R_{w}\right) / 2+\rho$ where $\rho$ is the expected number of passages between $v, w$ in $T$ steps. By Lemma 4 the number of light paths between $v, w$ is at most 2 . Using arguments similar to Lemma 9 , we find $\rho=O\left(T /(\delta-1)^{\omega^{\prime}}\right)=o(1 / \log n)$.

There is a natural measure-preserving mapping from the set of walks in $G$ which start at $u$ and do not visit $v$ or $w$, to the corresponding set of walks in $\widehat{G}$ which do not visit $Z$. Thus the probability that $\mathcal{W}_{u}$ does not visit $v$ or $w$ in steps $T$...t is asymptotically equal to the probability that a random walk $\widehat{\mathcal{W}}_{u}$ in $\widehat{G}$ which also starts at $u$ does not visit $Z$ in steps steps $T$...t. The detailed argument is given in [4].

We apply Lemma 2 to $\widehat{G}$. That $\pi_{Z}=\frac{2 d}{\theta n}$ is clear. Furthermore, the vertex $Z$ is tree-like up to distance $\omega$ in $\widehat{G}$. The derivation of $R_{Z}$ as in Lemma 9 (a) is valid. The fact that the root vertex of the corresponding infinite tree has degree $2 d$ does not affect the calculation of $R_{Z}^{*}$.

\section{References}

[1] R. Aleliunas, R.M. Karp, R.J. Lipton, L. Lovász and C. Rackoff, Random Walks, Universal Traversal Sequences, and the Complexity of Maze Problems. Proceedings of the 20th Annual IEEE Symposium on Foundations of Computer Science (1979) 218-223.

[2] B. Bollobás, A probabilistic proof of an asymptotic formula for the number of labelled regular graphs, European Journal on Combinatorics 1 (1980) 311-316.

[3] C. Cooper and A. M. Frieze. The cover time of random regular graphs, SIAM Journal on Discrete Mathematics, 18 (2005) 728-740.

[4] C. Cooper and A. M. Frieze. The cover time of the giant component of a random graph, Random Structures and Algorithms, 32 (2008) 401-439.

[5] D. Coppersmith, U. Feige, J. Shearer, Random walks on regular and irregular graphs, SIAM Journal on Discrete Mathematics, 9(2), 301-308, 1996. 
[6] U. Feige, A tight upper bound for the cover time of random walks on graphs, Random Structures and Algorithms, 6 (1995) 51-54.

[7] U. Feige, A tight lower bound for the cover time of random walks on graphs, Random Structures and Algorithms, 6 (1995) 433-438.

[8] U. Feige, Collecting coupons on trees, and the cover time of random walks, Computational Complexity, 6 (1996/1997), 341-356.

[9] B. McKay and N. Wormald, Asymptotic enumeration by degree sequence of graphs with degrees $o\left(n^{1 / 2}\right)$. Combinatorica, 11 (1991), 369-382.

[10] J.D. Kahn, N. Linial, N.Nisan and M.E. Saks, On the cover time of random walks on graphs. Journal of Theoretical Probability, 2(1):121-128 January 1989.

[11] A. Sinclair, Improved bounds for mixing rates of Markov chains and multicommodity flow. Combinatorics, Probability and Computing, 1 (1992), 351-370. 


\section{Appendix}

\section{Proof of conductance bound in Lemma 3}

By the conductance of a configuration $C$, we mean the conductance of a random walk on the underlying multi-graph $M(C)$. It is however, the configurations we sample uar in the proof of Lemma 10.

Lemma 10 Let $\boldsymbol{d}=\left(d_{1}, d_{2}, \ldots, d_{n}\right)$ be a sequence of natural numbers, satisfying $\min d_{i} \geq 3$ and $\theta \leq$ $n^{1 / 4}$. With probability $1-o\left(n^{-1 / 9}\right)$ the conductance $\Phi$ of a uar sampled configuration $\bar{C}(\boldsymbol{d})$ satisfies $\Phi \geq 0.01$.

Proof Let $F(a)=a ! /\left((a / 2) ! 2^{(a / 2)}\right)$. With this notation,

$$
\frac{F(b) F(a-b)}{F(a)}=\frac{\left(\begin{array}{l}
a / 2 \\
b / 2
\end{array}\right)}{\left(\begin{array}{l}
a \\
b
\end{array}\right)}=O(1)\left(\frac{b}{a}\right)^{b / 2}\left(1-\frac{b}{a}\right)^{(a-b) / 2} .
$$

For any $S \subseteq V$ let $d(S)$ denote the sum of the degrees of the vertices of $S$. A set $S$ is small if $d(S) \leq$ $(\theta n)^{1 / 4}$. A set is large if $(\theta n)^{1 / 4} \leq d(S) \leq \theta n / 2$. Let $\beta<1$ be a positive constant. We choose $\beta=0.99$. SMALL SETS $\left(\delta|S| \leq d(S) \leq(\theta n)^{1 / 4}\right)$.

Let $N(s, \beta)$ be the expected number of small sets $S$ of size $s$ with at least $\beta d(S)$ induced edges.

$$
N(s, \beta)=\sum_{S}\left(\begin{array}{c}
d(S) \\
\beta d(S)
\end{array}\right) \frac{F(\beta d(S)) F(\theta n-\beta d(S))}{F(\theta n)} .
$$

Thus using [30, $\delta s \leq d(s) \leq(\theta n)^{1 / 4}$ and $\delta \geq 3$ we find

$$
\begin{aligned}
N(s, \beta) & \leq O(1) \sum_{S}\left(\frac{e}{\beta}\right)^{\beta d(S)}\left(\frac{\beta d(S)}{\theta n}\right)^{\beta d(S) / 2} \\
& \leq O(1)\left(\frac{n e}{s}\left(\frac{e^{2}}{\beta(\theta n)^{3 / 4}}\right)^{3 \beta / 2}\right)^{s} \\
& =O\left(n^{-(9 \beta / 8-1) s}\right) .
\end{aligned}
$$

Thus

$$
\sum_{\substack{|S|=s \\ S \text { Small }}} N(s, \beta)=O\left(n^{-(9 \beta / 8-1)}\right) .
$$

LARGE $\operatorname{SETS}\left((\theta n)^{1 / 4} \leq d(S) \leq \theta n / 2\right)$.

Let $N(s, \beta)$ be the expected number of large sets $S$ of size $s$ inducing at least $\beta d(S)$ edges. As before, $N(s, \beta)$ is given by [31). Let $d(S)=\alpha \theta n$ where $0<\alpha \leq 1 / 2$. Let $\varepsilon=1-\beta$. We note the following approximation:

$$
\left(\begin{array}{c}
d(s) \\
\beta d(S)
\end{array}\right)=\left(\begin{array}{c}
\alpha \theta n \\
\beta \alpha \theta n
\end{array}\right)=\frac{O(1)}{\sqrt{\varepsilon \beta \alpha \theta n}} \frac{1}{\beta^{\beta \alpha \theta n} \varepsilon^{\varepsilon \alpha \theta n}} .
$$

Thus

$$
N(s, \beta) \leq \sum_{S} \frac{O(1)}{\sqrt{\varepsilon \beta \alpha \theta n}}\left(\frac{(\alpha \beta)^{\alpha \beta}(1-\alpha \beta)^{1-\alpha \beta}}{\left(\varepsilon^{\varepsilon} \beta^{\beta}\right)^{2 \alpha}}\right)^{\frac{\theta n}{2}}=\sum_{S} f(S) .
$$


Let $s=c n$. We henceforth assume that we choose the value $\alpha=\alpha^{*}$ which maximizes $f(S)$ for $|S|=c n$. With this convention we can write

$$
N(c n, \beta) \leq \frac{O(1)}{\sqrt{\varepsilon \beta c(1-c) \alpha \theta n^{2}}}\left(\left(\frac{(\alpha \beta)^{\alpha \beta}(1-\alpha \beta)^{1-\alpha \beta}}{\left(\varepsilon^{\varepsilon} \beta^{\beta}\right)^{2 \alpha}}\right)^{\frac{\theta}{2}} \frac{1}{c^{c}(1-c)^{1-c}}\right)^{n} .
$$

We split the proof for large sets into two parts: Those sets for which $\alpha \leq 1 / \theta$ and those for which $1 / \theta \leq \alpha \leq 1 / 2$.

Case of $\alpha \leq 1 / \theta$.

We need to remove the dependence on $c$ in the right hand side of the expression (33) for $N(c n, \beta)$. We first deal with the square root term. Since $\frac{1}{n} \leq c \leq \frac{(n-1)}{n}$, we have that $c(1-c) \geq \frac{n-1}{n^{2}}$ and so

$$
c(1-c) \alpha \theta n^{2} \geq \frac{n-1}{n^{2}}(\theta n)^{1 / 4} n \geq(\theta n)^{1 / 4} / 2 .
$$

Therefore, as $\beta, \varepsilon$ are positive constants,

$$
\frac{1}{\sqrt{\varepsilon \beta c(1-c) \alpha \theta n^{2}}}=\frac{O(1)}{(\theta n)^{1 / 8}} .
$$

We next consider the main term of 33. For $0 \leq x \leq 1 / 2$, the function

$$
g(x)=x^{x}(1-x)^{1-x}
$$

satisfies, $g(0)=1$ and is monotonically decreasing with minimum $g(1 / 2)=1 / 2$.

Since $d(S) \geq 3 s$, and $s=c n$, from $d(S)=\alpha \theta n$ we deduce that $c \leq \alpha \theta / 3$. As $\alpha \leq 1 / \theta$ then $c \leq \alpha \theta / 3 \leq 1 / 3$. Therefore $g(c) \geq g(\alpha \theta / 3)$, and we can replace $c$ by $\alpha \theta / 3$ in (33). Hence

$$
\begin{aligned}
N(c n, \beta) & =\frac{O(1)}{(\theta n)^{1 / 8}}\left(\frac{(\alpha \beta)^{\alpha \beta \theta / 2}(1-\alpha \beta)^{1-\alpha \beta \theta / 2}}{(\alpha \theta / 3)^{\alpha \theta / 3}(1-\alpha \theta / 3)^{1-\alpha \theta / 3}} \frac{(1-\alpha \beta)^{\theta / 2-1}}{\left(\varepsilon^{\varepsilon} \beta^{\beta}\right)^{\alpha \theta}}\right)^{n} \\
& =\frac{O(1)}{(\theta n)^{1 / 8}}(\phi(\alpha, \beta, \theta))^{n} .
\end{aligned}
$$

We next maximize $\phi(\alpha, \beta, \theta)$. Let $h(x, y)=(y x)^{x}(1-y x)^{1-x}$ for $0<x, y \leq 1$. Considering $h(x, y)$ as a function of $y$, there is a unique maximum at $y=1$, given by

$$
\begin{aligned}
\frac{\partial}{\partial y} \log (h(x, y)) & =x\left(\frac{1}{y}-\frac{1-x}{1-y x}\right)=0, \\
\frac{\partial^{2}}{\partial y^{2}} \log (h(x, y)) & =-x\left(\frac{1}{y^{2}}+\frac{x(1-x)}{(1-y x)^{2}}\right)<0 .
\end{aligned}
$$

Therefore $h(x, y)<h(x, 1)=g(x)$. So $h(\alpha \beta \theta / 2,2 / \theta)<g(\alpha \beta \theta / 2)<g(\alpha \theta / 3)$. Hence

$$
\phi(\alpha, \beta, \theta) \leq \frac{(1-\alpha \beta)^{\theta / 2-1}}{\left(\varepsilon^{\varepsilon} \beta^{\beta}\right)^{\alpha \theta}} .
$$


We prove below, that

$$
\frac{\partial}{\partial \theta}\left\{\frac{(1-\alpha \beta)^{\theta / 2-1}}{\left(\varepsilon^{\varepsilon} \beta^{\beta}\right)^{\alpha \theta}}\right\}<0
$$

Since $\theta \geq \delta \geq 3$, we have that

$$
\frac{(1-\alpha \beta)^{\theta / 2-1}}{\left(\varepsilon^{\varepsilon} \beta^{\beta}\right)^{\alpha \theta}} \leq \frac{e^{-\alpha \beta / 2}}{\left(\varepsilon^{\varepsilon} \beta^{\beta}\right)^{3 \alpha}} \leq \lambda^{\alpha},
$$

where $\lambda<0.7$, provided $\beta \geq 0.99$.

Now since $\alpha \theta n \geq(\theta n)^{1 / 4}$ for large sets, and $\theta \leq n^{1 / 4}$ by conditions of the lemma, we have that $\alpha n \geq n^{1 / 16}$. Thus

$$
\begin{aligned}
N(c n, \beta) & =\frac{O(1)}{(\theta n)^{1 / 8}}(\phi(\alpha, \beta, \theta))^{n} \\
& =O\left(\lambda^{n^{1 / 16}}\right)
\end{aligned}
$$

As $s=c n$ can take at most $n$ values we have that $\sum N(c n, \beta)=O\left(n \lambda^{n^{1 / 16}}\right)$.

Proof of 34.

$$
\frac{\partial}{\partial \theta}\left\{\frac{(1-\alpha \beta)^{\theta / 2-1}}{\left(\varepsilon^{\varepsilon} \beta^{\beta}\right)^{\alpha \theta}}\right\}=\frac{1}{1-\alpha \beta}\left(\frac{(1-\alpha \beta)^{\frac{1}{2}}}{\left(\varepsilon^{\varepsilon} \beta^{\beta}\right)^{\alpha}}\right)^{\theta} \log \left(\frac{(1-\alpha \beta)^{\frac{1}{2}}}{\left(\varepsilon^{\varepsilon} \beta^{\beta}\right)^{\alpha}}\right) .
$$

Let

$$
f(\alpha, \beta)=\frac{(1-\alpha \beta)}{\left(\varepsilon^{\varepsilon} \beta^{\beta}\right)^{2 \alpha}} .
$$

When $\alpha=0, f(\alpha, \beta)=1$. We prove that, for $\beta \geq 0.99, f(\alpha, \beta)<1$ for $\alpha>0$, which will establish the result. Note that

$$
\frac{\partial}{\partial \alpha} f(\alpha, \beta)=\frac{-1}{\left(\varepsilon^{\varepsilon} \beta^{\beta}\right)^{2 \alpha}}\left(\beta+(1-\alpha \beta) \log \left(\varepsilon^{\varepsilon} \beta^{\beta}\right)^{2}\right) .
$$

Consider

$$
\begin{aligned}
\frac{d}{d \beta}\left\{\log \left(\varepsilon^{\varepsilon} \beta^{\beta}\right)^{2}+\beta\right\} & \equiv \frac{d}{d \beta}\left\{\log \left((1-\beta)^{1-\beta} \beta^{\beta}\right)^{2}+\beta\right\} \\
& =2 \log \left(\frac{\beta}{1-\beta}\right)+1
\end{aligned}
$$

For $\beta>\frac{1}{2}$, the last line above is positive, and thus $\log \left(\varepsilon^{\varepsilon} \beta^{\beta}\right)^{2}>-\beta$. It follows that [35] is negative, as required.

Case of $1 / \theta \leq \alpha \leq 1 / 2$.

Continuing to evaluate $N(s, \beta)$ as before, and referring to $f(S)$ as given by the right hand side term of (32), let

$$
A(\alpha)=\frac{(\alpha \beta)^{\alpha \beta}(1-\alpha \beta)^{1-\alpha \beta}}{\left(\varepsilon^{\varepsilon} \beta^{\beta}\right)^{2 \alpha}} .
$$


Thus

$$
\begin{aligned}
\log (A(\alpha)) & =(\alpha \beta) \log ((\alpha \beta))+(1-\alpha \beta) \log (1-\alpha \beta)-2 \alpha \log \left(\varepsilon^{\varepsilon} \beta^{\beta}\right), \\
\frac{\partial}{\partial \alpha} \log (A(\alpha)) & =\beta \log (\alpha \beta)-\beta \log (1-\alpha \beta)-2 \log \left(\varepsilon^{\varepsilon} \beta^{\beta}\right) .
\end{aligned}
$$

Setting $\frac{\partial}{\partial \alpha} \log (A(\alpha))=0$ gives

$$
\alpha=\frac{\varepsilon^{2 \varepsilon / \beta} \beta}{1+\varepsilon^{2 \varepsilon / \beta} \beta^{2}} .
$$

Let $\alpha_{0}$ be the solution to this when $\beta=0.99$. Thus $\alpha_{0} \approx 0.477$. Also,

$$
\frac{\partial^{2}}{\partial \alpha^{2}} \log (A(\alpha))=\beta\left(\frac{1}{\alpha}+\frac{\beta}{1-\alpha \beta}\right)>0
$$

hence the stationary point $\alpha_{0}$ is a minima. As $\theta \geq 3$ and by inspection, $A(0.5)<A(1 / 3)$ then $A\left(\alpha_{0}\right) \leq$ $A(1 / \theta)$. We can use $\alpha^{*}=1 / \theta$ as the value of $\alpha$ maximizing $A(\alpha)$ in the range $1 / \theta \leq \alpha \leq 1 / 2$. It follows that

$$
\begin{aligned}
\sum_{\substack{S \operatorname{Large} \\
\alpha \geq 1 / \theta}} f(S) & =\left(\frac{1}{\sqrt{\theta n}}\right) 2^{n}(A(1 / \theta))^{\frac{\theta n}{2}} \\
& =O(1) 2^{n}\left(\frac{(\beta / \theta)^{\frac{\beta}{2}}(1-\beta / \theta)^{\frac{1}{2}(\theta-\beta)}}{\varepsilon^{\varepsilon} \beta^{\beta}}\right)^{n} .
\end{aligned}
$$

Let

$$
T(\theta)=\left(\frac{\beta}{\theta}\right)^{\beta}\left(1-\frac{\beta}{\theta}\right)^{\theta-\beta}
$$

then

$$
\frac{\partial}{\partial \theta} \log (T(\theta))=\log \left(\frac{\theta-\beta}{\theta}\right) .
$$

Thus $T(\theta)$ is monotone decreasing in $\theta$, and so $T(\theta) \leq T(3)$. Finally

$$
\begin{aligned}
\sum N(s, \beta) & \leq O(n) 2^{n}\left(\frac{(\beta / 3)^{\frac{\beta}{2}}(1-\beta / 3)^{\frac{1}{2}(3-\beta)}}{\varepsilon^{\varepsilon} \beta^{\beta}}\right)^{n} \\
& =O\left(n(0.8)^{n}\right) .
\end{aligned}
$$

This completes the proof of the lemma.

\section{Proof of Lemma 8}

For convenience, we restate the lemma.

Lemma 11 Let $\mathcal{W}_{v}^{*}$ denote the walk on $\Gamma_{v}$ starting at $v$ with $\Gamma_{v}^{\circ}$ made into absorbing states. Let $R_{v}^{*}=$ $\sum_{t=0}^{\infty} r_{t}^{*}$ where $r_{t}^{*}$ is the probability that $\mathcal{W}_{v}^{*}$ is at vertex $v$ at time $t$. There exists a constant $\zeta \in(0,1)$ such that

$$
R_{v}=R_{v}^{*}+O\left(\zeta^{\omega}\right)
$$


Proof We bound $\left|R_{v}-R_{v}^{*}\right|$ by using

$$
R_{v}-R_{v}^{*}=\left(\sum_{t=0}^{\omega} r_{t}-r_{t}^{*}\right)+\left(\sum_{t=\omega+1}^{T} r_{t}-r_{t}^{*}\right)-\sum_{t=T+1}^{\infty} r_{t}^{*} .
$$

Case $t \leq \omega$. When a particle starting from $v$ is absorbed at $\Gamma_{v}^{\circ}$, this is either at at distance $\omega$, or by a heavy vertex $u$ at distance less than $\omega$ from $v$. In the case of a heavy vertex $u$, by the light cycle condition, there are at most two light paths back to $v$ from $u$ of length at most $\omega$. All other paths of length at most $\omega$ go via other heavy vertices. Hence if a particle is at $u$, with probability at most $2 / \ell$ it will enter a light path to $v$. Thus the probability of reaching $v$ in time $\omega$ after having landed on a heavy vertex of $\Gamma_{v}^{\circ}$ is at most $O(\omega / \ell)=o\left(\zeta^{\omega}\right)$. In the alternative case that absorption is at distance $\omega$ from $v$, then for $t<\omega, r_{t}^{*}=r_{t}$. Thus we can write

$$
\left(\sum_{t=0}^{\omega} r_{t}-r_{t}^{*}\right)=o\left(\zeta^{\omega}\right)
$$

Case $\omega+1 \leq t \leq T$. Using 6 with $x=u=v$ and $\zeta=\left(1-\Phi^{2} / 2\right)<1$, we have for $t \geq \omega$, that $r_{t}=\pi_{v}+O\left(\zeta^{t}\right)$. Since $\Delta=O\left(n^{a}\right), a<1$, we have $T \pi_{v}=o\left(\zeta^{\omega}\right)$ and so

$$
\sum_{t=\omega+1}^{T}\left|r_{t}-r_{t}^{*}\right|=\sum_{t=\omega+1}^{T} r_{t} \leq \sum_{t=\omega+1}^{T}\left(\pi_{v}+\zeta^{t}\right)=O\left(\zeta^{\omega}\right) .
$$

Case $t \geq T+1$. It remains to estimate $\sum_{t=T+1}^{\infty} r_{t}^{*}$. We upper bound $r_{t}^{*}$ by a probability $\sigma_{t}$ as follows. Assume first that $\Gamma_{v}$ is a tree. Consider an unbiased random walk $X_{0}^{(b)}, X_{1}^{(b)}, \ldots$ starting at $|b|<a \leq \omega$ on the infinite line $(\ldots,-a, \ldots,-1,0,1, \ldots, a, \ldots) . X_{m}^{(b)}$ is the sum of $m$ independent \pm 1 random variables. The central limit theorem implies that there exists a constant $c>0$ such that

$$
\operatorname{Pr}\left(\left|X_{c a^{2}}^{(0)}\right|<a\right) \leq e^{-1 / 2} .
$$

Now for any $t$ and $b$ with $|b|<a$, we have

$$
\operatorname{Pr}\left(\left|X_{\tau}^{(b)}\right|<a, \tau=0, \ldots, t\right) \leq \operatorname{Pr}\left(\left|X_{\tau}^{(0)}\right|<a, \tau=0, \ldots, t\right)
$$

which is justified with the following game: We have two walks, $A$ and $B$ coupled to each other, with $A$ starting at position 0 and $B$ at position $b$, which, w.l.o.g, we shall assume is positive. The walk is a simple random walk which comes to a halt when either of the walks hits an absorbing state (that being, $-a$ or $a$ ). Since they are coupled, $B$ will win iff they drift $(a-b)$ to the right from 0 and $A$ will win iff they drift $-a$ to the left from 0 . Given the symmetry of the walk, $B$ has a higher chance of winning.

For $t>T$, we define $\sigma_{t}$ by

$$
\sigma_{t}=\operatorname{Pr}\left(\left|X_{\tau}^{(0)}\right|<a, \tau=0,1, \ldots, t\right) \leq\left(e^{-1 / 2}\right)^{\left\lfloor t /\left(c a^{2}\right)\right\rfloor} .
$$

The paths from $v$ to $\Gamma_{v}^{\circ}$ in the tree satisfy $a \leq \omega$, and so

$$
\sum_{t=T+1}^{\infty} \sigma_{t} \leq \sum_{t=T+1}^{\infty} e^{-t /\left(3 c \omega^{2}\right)} \leq \frac{e^{-T /\left(3 c \omega^{2}\right)}}{1-e^{-1 /\left(3 c \omega^{2}\right)}}=O\left(\omega^{2} e^{-\Theta\left(\frac{\log n}{\omega^{2}}\right)}\right)=O\left(\zeta^{\omega}\right)
$$


We now turn to the case where $\Gamma_{v}$ contains a unique light cycle $C$. Let $x$ be the furthest vertex of $C$ from $v$ in $\Gamma_{v}$. This is the only possible place where the random walk is more likely to get closer to $v$ at the next step. We can see this by considering the breadth first construction of $\Gamma_{v}$. Thus we can compare our walk with random walk on $[-a, a]$ where there is a unique value $x<a$ such that only at $\pm x$ is the walk more likely to move towards the origin and even then this probability is at most 2/3. Using results 39], 40] for the unbiased walk on the line, we have

$$
\operatorname{Pr}\left(\exists \tau \leq c a^{2}:\left|X_{\tau}^{(b)}\right| \geq x\right) \geq 1-e^{-1 / 2} .
$$

The probability the particle walks from $x$ to $a$ without returning to the cycle is at least $1 / 3(a-x)$. Thus

$$
\operatorname{Pr}\left(\exists \tau \leq c a^{2}:\left|X_{\tau+a-x}^{(b)}\right| \geq a\right) \geq\left(1-e^{-1 / 2}\right) / 3 a \geq \frac{13}{100 a},
$$

and so

$$
\sigma_{t}=\operatorname{Pr}\left(\left|X_{\tau}^{(0)}\right|<a, \tau=0,1, \ldots, t\right) \leq(1-13 /(100 a))^{\left\lfloor t /\left(2 c a^{2}\right)\right\rfloor} \leq e^{-t /\left(20 c a^{3}\right)} .
$$

As $a \leq \omega$,

$$
\sum_{t=T+1}^{\infty} \sigma_{t} \leq \sum_{t=T+1}^{\infty} e^{-t /\left(20 c \omega^{3}\right)} \leq \frac{e^{-T /\left(20 c \omega^{3}\right)}}{1-e^{-1 /\left(20 c \omega^{3}\right)}}=O\left(\omega^{3} e^{-O\left(\frac{\log n}{\omega^{3}}\right)}\right)=O\left(\zeta^{\omega}\right)
$$

\section{Condition (a) of Lemma2}

Lemma 12 There exists a constant $\psi>0$ such that for $|z| \leq 1+\lambda,\left|R_{T}(z)\right| \geq \psi$.

Proof As in Lemma 8 , we consider the walk $\mathcal{W}_{v}^{*}$ on $\Gamma_{v}$, starting from $v$, and with absorption at $\Gamma_{v}^{\circ}$. For this walk, let $\beta_{t}$ be the probability of a first return to $v$ at step $t$, and let $r_{t}^{*}$ be the probability of a return to $v$ at step $t$.

Let $\beta(z)=\sum_{t=1}^{T} \beta_{t} z^{t}$, let $\alpha(z)=1 /(1-\beta(z))$, and write $\alpha(z)=\sum_{t=0}^{\infty} \alpha_{t} z^{t}$. Thus $\alpha_{t}$ is the probability of a return to $v$ at time $t$ for a walk $\mathcal{W}_{v}^{\dagger}$, all of whose excursions from $v$ are length at most $T$. Observe that $\alpha_{t} \leq r_{t}^{*} \leq r_{t}$. We shall prove below that the radius of convergence of $\alpha(z)$ is at least $1+\Omega\left(1 / \omega^{3}\right)$.

We can write

$$
\begin{aligned}
R_{T}(z) & =\alpha(z)+Q(z) \\
& =\frac{1}{1-\beta(z)}+Q(z),
\end{aligned}
$$

where $Q(z)=Q_{1}(z)+Q_{2}(z)$, and

$$
\begin{aligned}
& Q_{1}(z)=\sum_{t=0}^{T}\left(r_{t}-\alpha_{t}\right) z^{t} \\
& Q_{2}(z)=-\sum_{t=T+1}^{\infty} \alpha_{t} z^{t} .
\end{aligned}
$$


We note that $Q(0)=0, \alpha(0)=1$ and $\beta(0)=0$.

We claim that the expression (43) is well defined for $|z| \leq 1+\lambda$. We will show below that

$$
\left|Q_{2}(z)\right|=o(1)
$$

for $|z| \leq 1+2 \lambda$ and thus the radius of convergence of $Q_{2}(z)$ (and hence $\alpha(z)$ ) is greater than $1+\lambda$. This will imply that $|\beta(z)|<1$ for $|z| \leq 1+\lambda$. For suppose there exists $z_{0}$ such that $\left|\beta\left(z_{0}\right)\right| \geq 1$. Then $\beta\left(\left|z_{0}\right|\right) \geq\left|\beta\left(z_{0}\right)\right| \geq 1$ and we can assume (by scaling) that $\beta\left(\left|z_{0}\right|\right)=1$. We have $\beta(0)<1$ and so we can assume that $\beta(|z|)<1$ for $0 \leq|z|<\left|z_{0}\right|$. But as $\rho$ approaches 1 from below, 43 is valid for $z=\rho\left|z_{0}\right|$ and then $\left|R_{T}\left(\rho\left|z_{0}\right|\right)\right| \rightarrow \infty$, contradiction.

Recall that $\lambda=1 / K T$. Clearly $\beta(1) \leq 1$ and so for $|z| \leq 1+\lambda$

$$
\beta(|z|) \leq \beta(1+\lambda) \leq \beta(1)(1+\lambda)^{T} \leq e^{1 / K} .
$$

Using $|1 /(1-\beta(z))| \geq 1 /(1+\beta(|z|))$ we obtain

$$
\left|R_{T}(z)\right| \geq \frac{1}{1+\beta(|z|)}-|Q(z)| \geq \frac{1}{1+e^{1 / K}}-|Q(z)| .
$$

We now prove that $|Q(z)|=o(1)$ for $|z| \leq 1+\lambda$ and the lemma will follow.

Turning our attention first to $Q_{1}(z)$, we have

$$
\left|Q_{1}(z)\right| \leq(1+\lambda)^{T}\left|Q_{1}(1)\right| \leq e^{2 / K} \sum_{t=0}^{T}\left|r_{t}-\alpha_{t}\right| .
$$

From (37), (38) of the proof of Lemma 8 we see that $\sum_{t=0}^{T}\left|r_{t}-\alpha_{t}\right|=o(1)$, hence $\left|Q_{1}(z)\right|=o(1)$.

We now consider $Q_{2}(z)$. As in Lemma 8 , let $r_{t}^{*}$ be the probability that a walk $\mathcal{W}_{v}^{*}$ on $\Gamma_{v}$ starting at $v$ has not been absorbed at $\Gamma_{v}^{\circ}$ by step $t$. Then $\alpha_{t} \leq r_{t}^{*} \leq \sigma_{t}$, so

$$
\left|Q_{2}(z)\right| \leq \sum_{t=T+1}^{\infty} \sigma_{t}|z|^{t}
$$

In the case where $G_{v}$ is a tree we can use 41] to prove that the radius of convergence of $Q_{2}(z)$ is at least $e^{1 /\left(3 c \omega^{2}\right)} \gg 1+2 \lambda$. So for $|z| \leq 1+\lambda$,

$$
\left|Q_{2}(z)\right| \leq \sum_{t=T+1}^{\infty} e^{\lambda t-t /\left(3 c \omega^{2}\right)}=o(1)
$$

In the case that $G_{v}$ contains a unique cycle, we can use the see that the radius of convergence of $Q_{2}(z)$ is at least $e^{\frac{1}{20 c \omega^{3}}} \gg 1+2 \lambda$. So for $|z| \leq 1+\lambda$,

$$
\left|Q_{2}(z)\right| \leq \sum_{t=T+1}^{\infty} e^{\lambda t-t /\left(20 c \omega^{3}\right)}=o(1) .
$$


